Journal de la Société des américanistes

Journal de la Société

des américanistes

tome $93, \mathrm{n}^{\circ} 1$

\title{
The Ancient Maya et la Stèle «E » de Quiriguá
}

André Segura

\section{CpenEdition}

Journals

Édition électronique

URL : https://journals.openedition.org/jsa/6973

DOI : 10.4000/jsa.6973

ISSN : $1957-7842$

Éditeur

Société des américanistes

Édition imprimée

Date de publication : 1 décembre 2007

Pagination : 164-174

ISSN : 0037-9174

\section{Référence électronique}

André Segura, «The Ancient Maya et la Stèle «E » de Quiriguá », Journal de la Société des américanistes

[En ligne], 93-1 | 2007, mis en ligne le 15 juin 2012, consulté le 03 septembre 2022. URL : http:// journals.openedition.org/jsa/6973; DOI : https://doi.org/10.4000/jsa.6973 


\title{
NOTES DE RECHERCHE
}

\section{THE ANCIENT MAYA ET LA STÈLE « E » DE QUIRIGUÁ}

\author{
André SEGURA *
}

La lecture de la cinquième et de la sixième éditions de The Ancient Maya (Sharer 1994 ; Sharer et Traxler 2006) pose un certain nombre de problèmes dont je ne voudrais évoquer ici que celui de l'attribution à la Stèle F de Quiriguá (ou « Monument 6 » dans la terminologie du Quirigua Project : Sharer et Ashmore 1979) de la Série Initiale 9.17.0.0.0 13 Ahau 18 Cumkú. L'identification de ce problème renvoie à un autre, beaucoup plus sérieux, qui concerne, lui, le dessin publié dans les éditions antérieures de l'ouvrage. Le problème de l'attribution se pose à la comparaison des trois premières éditions de The Ancient Maya (Morley 1946, 1947; Morley et Brainerd 1956) et des deux dernières éditions (Sharer 1994 ; Sharer et Traxler 2006). En effet, les titres qui accompagnent le dessin de la Série Initiale mentionnée se réfèrent à la face Est de la Stèle $\mathrm{E}$ dans les trois premières éditions, mais à face Est du Monument 6 ou Stèle $\mathrm{F}$ pour les éditions Sharer (1994) et Sharer et Traxler (2006). Pour trancher ce dilemme, il suffit de s'en remettre à des photographies des faces Est des deux stèles (Figures 1 et 2). Leur comparaison fait ressortir que les deux faces Est portent deux dates différentes, libellées toutes deux dans le système du Compte Long. Leurs différences ne se limitent pas au jour enregistré ${ }^{1}$, mais ont trait également à la forme des coefficients numériques affectant les unités de temps : sur la Stèle F, ces coeffïcients ont une forme anthropomorphique, alors que sur la Stèle E ils sont écrits dans le système de barres et points. La confusion des deux stèles n'est pas possible : Sharer a donc commis une erreur (à moins qu'elle ne soit due au typographe ${ }^{2}$ ), et nombre de mayanistes, oubliant Morley, ont contribué, de bonne foi, à la propager ${ }^{3}$.

L'erreur du titre étant signalée, il reste le problème du dessin. On observe que le même dessin apparaît dans toutes les éditions de The Ancient Maya ; mais la

\footnotetext{
• Université du Sud-Toulon-Var, avenue de l'Université, BP 20132, 83957 La Garde [segura.andre (a)wanadoo.fr].
}

Journal de la Société des Américanistes, 2007, 93-1, pp. 167-174. O Société des Américanistes. 


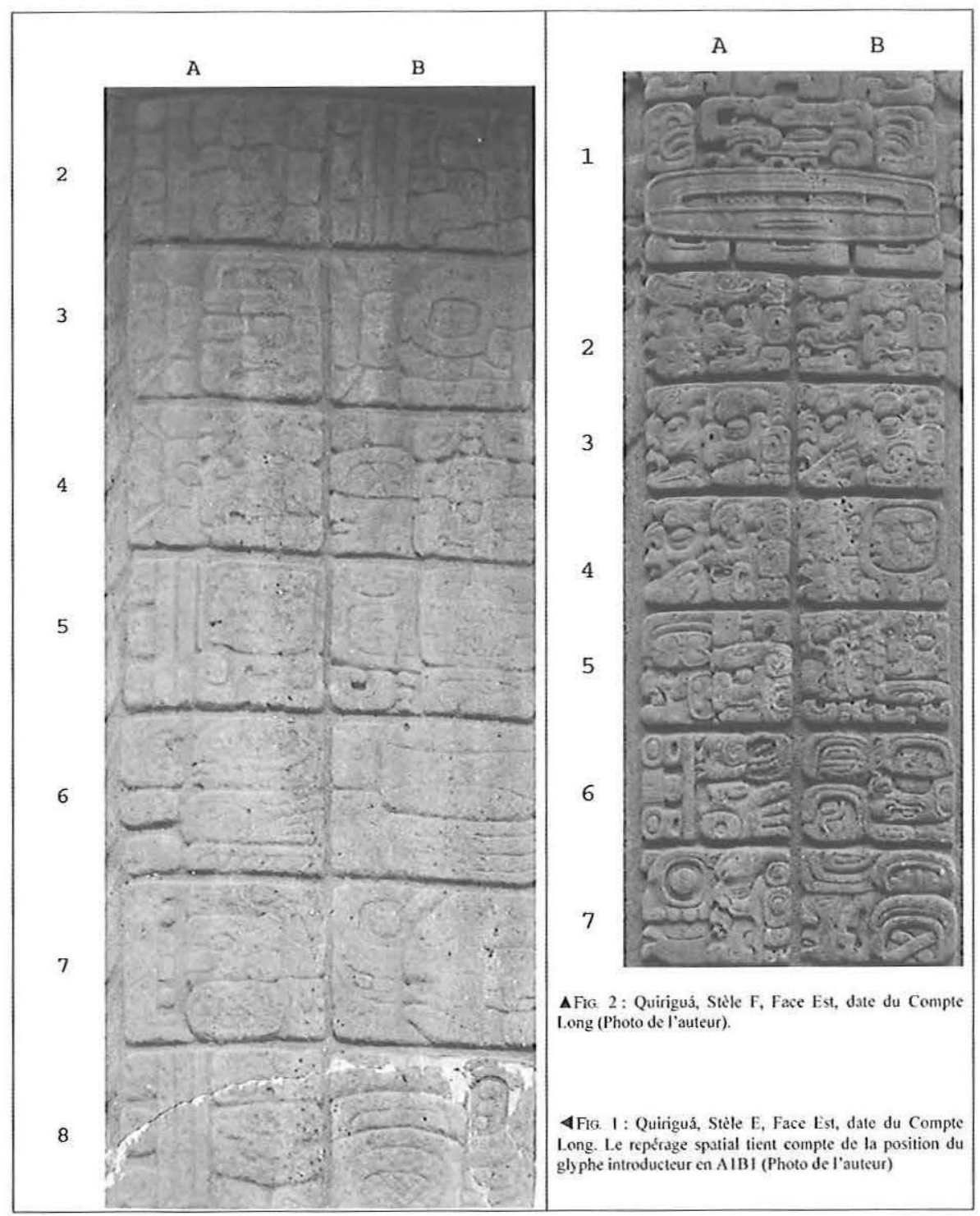


mise en parallèle dudit dessin et de la photographie de la face Est de la Stèle E révèle que le premier n'est pas conforme à la réalité dont il prétend donner une image. Les différences entre la Série Initiale sculptée sur la face Est de la Stèle E et le dessin de Morley (Figure 4) portent sur trois points :

1) Les deux glyphes G9 et F du dessin de Morley, réunis sur la stèle originale, y occupent ensemble la position B4, place assignée à la référence Tzolkín (13 Ahau) dans le dessin.

2) Les deux glyphes G9 et F occupent donc deux positions séparées dans le dessin, respectivement A5 et B5, alors que, sur la sculpture, A5 est la position occupée par la référence Tzolkín.

3) Du fait sans doute de la séparation des glyphes $G$ et $F$, la référence Haab (18 Cumkú) passe de la position A8 qu'elle occupe vraiment sur la stèle à la position B8 dans le dessin.

Finalement l'erreur de Sharer est une «bonne erreur » car elle a permis de (re)découvrir que Morley avait donné de la Série Initiale de la face Est de la Stèle E une reproduction graphique modifiée. Il reste à établir les raisons qui ont pu pousser ce dernier à introduire de telles modifications.

Des éléments de réponse sont fournis en des termes identiques dans les deux premières éditions de The Ancient Maya, dans lesquelles on peut lire, au début du commentaire relatif au dessin : " a brief description of a typical Initial Series follows » (Morley 1946, p. 285) ; puis, plus loin :

The day of this terminal date - here "13 Ahau" - is usually found (nine out of ten times) in the sixth position after the introducing-glyph, or immediately following the fifth and last time period (the kins) of the Initial Series number (Fig. 25). In almost all cases, the glyph immediately following the day of Initial Series terminal date, the one in the seventh position after the large introducing-glyph (Fig. 25) is called Glyph G... (Morley 1946, pp. 287-288).

À la lecture de ces phrases, il semble bien que Morley ait voulu offrir, avec le dessin modifié, un modèle général des dates écrites dans le système du Compte Long. On doit d'ailleurs remarquer que la volonté de représenter graphiquement un modèle général de Série Initiale est présent dès le Guía de las ruinas de Quiriguá (Morley 1936, p. 216) pour lequel fut initialement conçu le dessin rattaché plus tard à la Stèle E dans The Ancient Maya. Mais, dans le premier ouvrage, le dessin n'est présenté à aucun moment comme étant la reproduction graphique d'une Série Initiale réelle, pas plus à Quiriguá qu'ailleurs (ibid., pp. 215-218), même si l'on peut penser qu'il fut inspiré par la Série Initiale de la face Est de Stèle E. Ce dessin est en effet donné dans le Guía de las ruinas... comme la représentation graphique d'une Série Initiale "typique » ${ }^{4}$, dont il faut supposer qu'elle est fictive. C'est en passant du Guía de las ruinas... à The Ancient Maya que le dessin change de statut : illustration d'une Série Initiale fictive dans le premier, il devint 


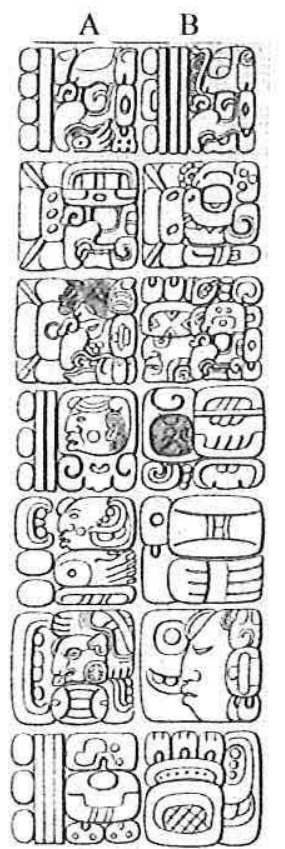

Fig. 3: Quiriguá, Stèle E, Cỏté Est, reproduction graphique de La Série Initiale (et de la Série Supplémentaire) (Maudslay, 1899-1902).
2

3

4

5

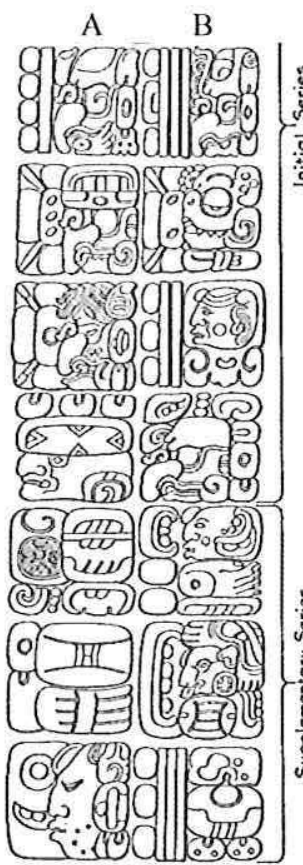

\begin{tabular}{|c|c|}
\hline $\begin{array}{l}9 \times 144,000 \text { diys } \\
=1,295,000 \text { diys) }\end{array}$ & 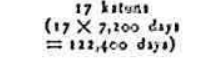 \\
\hline $\begin{array}{l}\text { o teas } \\
(0 \times 360 d a y t \\
=0 \text { diyi) }\end{array}$ & $\begin{array}{l}\text { ovinalo } \\
(0 \times 20 \text { diys } \\
=0<2 y i)\end{array}$ \\
\hline 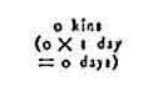 & 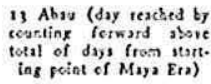 \\
\hline
\end{tabular}

\section{Glysh G?}

Nameralyph of the detity who is pstron of the Nint Glych $F$ (The Nine G-ds of the the Loate Wot

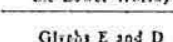

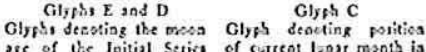

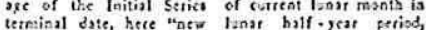

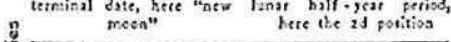

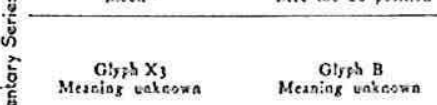

Flg 4: Quiriguá, Stèle E, Còté Est, prétendue reproduction graphique de La Série Initiale (et de la Série Supplémentaire) (Morley, 1946)

représentation graphique d'une Série Initiale réelle dans le second ${ }^{5}$. On est surpris par la liberté prise par Morley, d'autant qu'il existait un dessin de Maudslay (1899-1902, vol. 5, p. 40) de cette face de la stèle (Figure 3), conforme, lui, à la réalité.

Mais d'autres changements sont encore visibles d'un livre à l'autre. Dans le Guía de las ruinas..., Morley (1936, p. 218) considérait le Glyphe G9 comme le premier de la Série Supplémentaire, et c'est là probablement une des deux raisons qui l'amenèrent à permuter, dans son dessin, la référence Tzolkín et le Glyphe G9, puisque cette permutation permettait d'isoler la Série Supplémentaire de la Série Initiale. Cette première raison disparut dès la première édition de The Ancient Maya, car le Glyphe G9 y apparaît intégré à la Série Initiale. Mais la deuxième raison, qui justifiait aussi, et sans doute davantage, la permutation opérée, demeurait et, avec elle, cette dernière. Aux yeux de Morley, en effet, la position de la référence Tzolkín était atypique sur la stèle, voire erronée ${ }^{6}$, en comparaison avec les Séries Initiales, jumelle, de la face Ouest de la Stèle E et, cousine, de la Stèle $\mathrm{F}^{7}$ du même site. Cette « anomalie », qui différenciait la Série Initiale de la 
face Est de la Stèle E de ces dernières, ne la rapprochait pas pour autant de stèles connues d'autres sites et aurait donc empêché le dessin qui l'aurait reproduite d'accéder au statut de "modèle général ». Dans le même temps, une autre « anomalie » existait concernant la position du Glyphe G par rapport aux Séries Initiales des Stèles F et E/Ouest. Il fallait donc que Morley s'écartât de sa source d'inspiration (la face Est de la Stèle E) sur les deux positions de la référence Tzolkín et du Glyphe G, s'il prétendait présenter un modèle général de Série Initiale. Ayant permuté ces positions, il pensait sans doute avoir établi un modèle dont la portée dépassait les limites de Quiriguá ${ }^{8}$.

Un indice de ce qu'avec la représentation graphique modifiée Morley prétendait offrir un schéma général transparaît dans son mode de repérage de la position des glyphes : celle-ci est donnée par leur rang après le glyphe introducteur (la référence Tzolkín est « in the sixth position after the introducingglyph » et le Glyphe $\mathrm{G}$ occupe " the seventh position after the large introducingglyph "; Morley 1936, pp. 217-218; 1946; 1947, pp. 287-288), et non par rapport à un système d'axes portant des lettres en abscisses et des nombres en ordonnées. De la sorte, le modèle de Série Initiale était toujours applicable, que celle-ci ait été disposée en une ou deux colonnes. Une des conditions était ainsi créée pour que la Série Initiale de la Plaque de Leyden, la plus ancienne connue, au moment où les deux premières éditions de The Ancient Maya furent publiées (Morley 1946; 1947, p. 43), puisse être considérée comme conforme au modèle bien que formée d'une seule colonne jusqu'à la référence Tzolkín. Ce à quoi tenait sans doute beaucoup Morley. Le second élément à prendre en compte était que les considérations sur le Glyphe G ne concernent pas le Glyphe F, lequel est associé au premier sur la face Est de la Stèle E : de fait, dans la Série Initiale de la Plaque de Leyden, le Glyphe F est absent. Le règlement de ce problème fut obtenu, après la permutation signalée, par la séparation des deux Glyphes $\mathrm{G}$ et $\mathrm{F}$ qui occupèrent de ce fait les positions $\mathrm{A} 5$ et $\mathrm{B} 5$ dans le dessin.

Le dessin s'appliquant à des Séries Initiales sculptées à des périodes différentes (y compris à la première d'entre elles) et sous des formes différentes pouvait vraiment être considéré comme l'incarnation d'un modèle général.

La séparation des Glyphes $\mathrm{G}$ et $\mathrm{F}$ eut pour résultat de renforcer le statut de modèle général du dessin par une autre voie. En effet, cette dissociation, toutes choses égales par ailleurs, aboutit à repousser la référence Haab de $\mathrm{A} 8$, position qu'elle occupe réellement sur la Stèle E, en B8. Ce faisant, dans le dessin, les deux références se situent dans la même colonne comme sur la stèle donc, à ceci près que, sur cette dernière, il s'agit de la colonne A alors que, dans le dessin, il s'agit de la colonne B. Cette analyse semble indiquer qu'il y allait là aussi du poids du modèle, puisque l'on constate que c'est justement dans la colonne B que sont situées les références Tzolkín et Haab des Séries Initiales de la Stèle E (face Ouest) et de la Stèle F (face Est). À cette dernière observation, il pourrait être objecté que, 
dans la Série Initiale de la face Est de la Stèle C, les deux références Tzolkín et Haab n'appartiennent pas à la même colonne (on les trouve en B4 et A5). Mais Morley avait parfaitement pu compter la Stèle C parmi les exceptions (par rapport à « la norme » du dessin) puisqu'il écrivait, à propos du Glyphe G, que " in almost all cases », ce dernier occupait la " the seventh position after the large introducing-glyph » (ibid.).

L'ensemble de ces considérations amène à s'interroger finalement sur ce qui a pu pousser Morley à préférer construire son modèle général en modifiant la Série Initiale de la face Est de la Stèle E, plutôt qu'en reproduisant fidèlement celle de la face Ouest de la même Stèle ${ }^{9}$ ou celle de la face Est de la Stèle F. Rappelons que, dans ces deux dernières, la référence Tzolkín est en B4, le Glyphe G en A5, tandis que la référence Haab occupe une position située dans colonne B.

Les Séries Initiales étant, pour Morley (1936, pp. 181-182 ; 1946, p. 262), l'illustration par excellence de l'utilisation des nombres, on peut penser que celui-ci a voulu représenter une Série Initiale contenant tous les éléments constitutifs du système de numération. Cela imposait de choisir une Série Initiale dont les coefficients numériques étaient rédigés dans le système barres et points (les cinq unités de temps d'une telle série ne permettent évidemment pas la représentation des quatorze têtes appartenant à la figuration anthropomorphe des nombres, de 0 à 13, et du même coup, les Séries Initiales de la Stèle F, avec leurs nombres en variantes de têtes, ne pouvaient pas être retenues comme sources du modèle à dessiner). Par ailleurs, les nombres écrits dans le système barres et points étant d'une lecture plus aisée que ceux qui le sont dans le système anthropomorphe, le degré de facilité de lecture a dû jouer le rôle d'adjuvant dans le choix d'une Série Initiale rédigée dans le système barres et points avec en plus le zéro, ce qui excluait la face Ouest de la Stèle E.

La prétention à donner à voir un modèle général, confirmée au niveau du texte des deux premières éditions de The Ancient Maya, fut toutefois brouillée parce que le dessin artificiel, préparé pour le Guia de las ruinas..., fut présenté alors comme la reproduction graphique de la Série Initiale et de la Série Supplémentaire de la face Est de la Stèle E, qualifiées dans le titre de " examples ». Dans la troisième édition, revue par Brainerd, tandis que l'attribution du dessin à la Stèle E fut maintenue, le texte abandonna certaines des considérations généralisatrices des deux premières éditions ("A brief description of a typical Initial Series follows"; " usually... (nine out of ten times) 》; " in almost all cases »). Dans la troisième édition, le commentaire qui accompagne le dessin présenté comme étant celui de la face Est de la Stèle E n'insiste plus autant sur les principes de présentation d'une Série Initiale qui auraient été valables de façon générale.

Si l'on considère aujourd'hui qu'un modèle général n'est plus concevable, ne faudrait-il pas revenir au dessin de Maudslay (Figure 3) de telle sorte que la représentation graphique de la face Est de la Stèle E donne de la Série Initiale 
qu'elle porte une image fidèle ? Mais serait-il pertinent de proposer au lecteur (de la septième édition de The Ancient Maya ?) comme illustration d'une Série Initiale un exemple dans lequel la référence Tzolkín et le Glyphe G occupent des positions peu communes? *

* Manuscrit reçu en novembre 2006, accepté pour publication en janvier 2007.

\section{NOTES}

1. Pour la face Est de la Stèle F, il s'agit du jour 9.16.10.0.0 I Ahau 3 Zip.

2. C'est certainement l'hypothèse qu'il convient de privilégier, puisque Sharer reproduit par ailleurs la photographie de Morley (1947, p. 286) de la face Est de la Stèle F (voir Sharer 1994, p. 652).

3. Demarest (2004, p. 197) ne doit pas être compté au nombre des propagateurs puisqu'il reproduit, lui, le dessin d'A. P. Maudslay de la face Est de la Stèle F et l'affecte bien à ladite stèle.

4. Légende du dessin : "Series inicial y suplementaria tipicas, con su explicación 》 (Morley 1936, p. 217).

5. Légende du même dessin que dans le Guia de las ruinas... : " Examples of an initial and a supplementary series : east side of Stela E, Quirigua ” (Morley 1946, p. 286).

6. Le texte de la Stèle E est porteur de quatre erreurs selon lui (Morley 1946, Tableau XI, en face de la page 448 ).

7. Ou parentes éloignées telles que celles des faces Est des Stèles $A$ et $C$ pour ce qui concerne la seule position de la référence Tzolkín.

8. Dont, néanmoins, plusieurs stèles de Quiriguá même s'écartent. Ainsi, alors que, sur le modèle général, le Glyphe G se trouve en position A5, sur la face Nord de la Stèle K, la référence Tzolkín et le Glyphe G9 ensemble occupent la position A4 (parce que les uinals et les kins sont contigus en B3). Si la référence Tzolkín occupe bien la position B4 sur la face Est de la Stèle C, c'est la référence Haab qui est en A5 et non le Glyphe G. Enfin, sur la face Est de la Stèle A, le Glyphe G suit bien la référence Tzolkín située en B4, mais en B5 et non en A5.

9. En corrigeant, néanmoins, l'erreur affectant le nombre de tuns.

\section{RÉFÉRENCES BIBLIOGRAPHIQUES}

Demarest Arthur A.

2004 Ancient Maya. The rise and fall of a rainforest civilization, Cambridge University Press, Cambridge.

Maudslay Alfred Percival

1899-1902 Biologia Centrali-Americana : archaeology, 6 volumes, R. H. Porter et Dulau \& Co, Londres [édition facsimilé, Milpatron Publishing Corp, New York, 1974].

MORLey Sylvanus G.

1936 Guía de las ruinas de Quiriguá, Institución Carnegie de Washington, Washington [traduction du Guide book to the Ruins of Quirigua, Carnegie Instution of Washington Supplemental Publication n ${ }^{\circ} 16$, Washington, DC, 1935]. 
1946 The Ancient Maya, Stanford University Press, Stanford, California [1 ${ }^{\text {re }}$ édition].

1947 The Ancient Maya, Stanford University Press, Stanford, California [2 édition].

Morley Sylvanus G. et George W. Brainerd

1956 The Ancient Maya, Stanford University Press, Stanford, California [ $3^{\mathrm{e}}$ édition].

SHARER Robert J.

1994 The Ancient Maya, Stanford University Press, Stanford, California [5e édition].

Sharer Robert J. et Wendy Ashmore

1979 Quirigua reports, vol. 1, The University Museum, University of Pennsylvania, Philadelphie.

SHARER Robert J. et Loa P. Traxler

2006 The Ancient Maya, Stanford University Press, Stanford, California [6 édition]. 